\title{
SARS-CoV-2 viroporin triggers the NLRP3 inflammatory pathway
}

4 Authors: Huanzhou Xu, ${ }^{1}$ Siddhi A. Chitre, ${ }^{1}$ Ibukun A. Akinyemi, ${ }^{2}$ Julia C. Loeb, ${ }^{3,4}$ John A.

5 Lednicky, ${ }^{3,4}$ Michael T. McIntosh, ${ }^{2,5}$ and Sumita Bhaduri-McIntosh ${ }^{1,5, *}$

7 Affiliations:

$8{ }^{1}$ Division of Infectious Disease, Department of Pediatrics, University of Florida, Gainesville, FL, 9 U.S.A.

$10{ }^{2}$ Child Health Research Institute, Department of Pediatrics, University of Florida, Gainesville, FL, 11 U.S.A.

$12{ }^{3}$ Department of Environmental and Global Health, College of Public Health and Health 13 Professions, University of Florida, Gainesville, FL, U.S.A.

$14{ }^{4}$ Emerging Pathogens Institute, University of Florida, Gainesville, FL, U.S.A.

$15{ }^{5}$ Department of Molecular Genetics and Microbiology, University of Florida, Gainesville, FL, U.S.A.

17

* To whom correspondence should be addressed at sbhadurimcintosh@ufl.edu 


\section{Abstract}

26 Cytokine storm resulting from a heightened inflammatory response is a prominent feature of severe

27 COVID-19 disease. This inflammatory response results from assembly/activation of a cell-

28 intrinsic defense platform known as the inflammasome. We report that the SARS-CoV-2 viroporin

29 encoded by ORF3a activates the NLRP3 inflammasome, the most promiscuous of known

30 inflammasomes. ORF3a triggers IL-1 $\beta$ expression via $\mathrm{NF} \kappa \mathrm{B}$, thus priming the inflammasome

31 while also activating it via ASC-dependent and -independent modes. ORF3a-mediated

32 inflammasome activation requires efflux of potassium ions and oligomerization between NEK7

33 and NLRP3. With the selective NLRP3 inhibitor MCC950 able to block ORF3a-mediated

34 inflammasome activation and key ORF3a residues needed for virus release and inflammasome

35 activation conserved in SARS-CoV-2 isolates across continents, ORF3a and NLRP3 present prime

36 targets for intervention.

37 


\section{Summary}

47 Development of anti-SARS-CoV-2 therapies is aimed predominantly at blocking infection or

48 halting virus replication. Yet, the inflammatory response is a significant contributor towards

49 disease, especially in those severely affected. In a pared-down system, we investigate the influence

50 of ORF3a, an essential SARS-CoV-2 protein, on the inflammatory machinery and find that it

51 activates NLRP3, the most prominent inflammasome by causing potassium loss across the cell

52 membrane. We also define key amino acid residues on ORF3a needed to activate the inflammatory

53 response, and likely to facilitate virus release, and find that they are conserved in virus isolates

54 across continents. These findings reveal ORF3a and NLRP3 to be attractive targets for therapy.

55

56

57

58

59

60

61

62

63

64

65 


\section{Main Text}

67 Worldwide reports of COVID-19 indicate that effective management of severely ill individuals

68 will require both antiviral and anti-inflammatory strategies. Indeed, during the second week of

69 illness, those with severe disease experience cytokine storms indicating a massive inflammatory

70 surge ${ }^{1,2}$. This inflammatory response, composed of IL-1 $\beta$ and other cytokines, results from

71 assembly/activation of a multiprotein host machinery known as the inflammasome in both immune

72 and non-immune cells such as airway epithelial cells - the most prominent is the NLRP3 (NOD-,

73 LRR- and pyrin domain-containing protein 3)-inflammasome - and several lines of evidence tie

74 activation of the NLRP3-inflammasome to severe SARS-CoV-2 pathology, including i)

75 individuals with comorbidities such as diabetes, atherosclerosis, and obesity (all pro-inflammatory

76 conditions marked by NLRP3 activation) ${ }^{3-8}$ are at greater risk for severe disease ${ }^{9-11}$, ii) viroporins

77 expressed by the closely-related SARS-CoV activate the NLRP3 inflammasome ${ }^{12}$, and iii) bats,

78 the asymptomatic reservoir of CoVs that are highly pathogenic in humans, are naturally defective

79 in activating the NLRP3-inflammasome ${ }^{13}$. Although cellular ACE2 engagement by SARS-CoV-

802 spike protein can cause expression of pro-inflammatory genes ${ }^{14}$, whether CoV-2 activates the

81 inflammasome remains unexplored.

83 Given the central role of inflammation in severe COVID-19 and the high level of conservation of

84 the viroporin ORF3a across CoV genomes, we investigated the influence of the SARS-CoV-2

85 ORF3a on the NLRP3 inflammasome. Viroporins are virus-encoded proteins that are considered

86 virulence factors. Though typically not essential for virus replication, some of these small

87 hydrophobic proteins can form pores that facilitate ion transport across cell membranes, and by so

88 doing, ensure virus release with the potential for coincident inflammasome activation ${ }^{15,16}$. A 
component of the innate immune system, the inflammasome assembles and responds to invading

90

91

92

93

94

organisms, thus forming the first line of defense against infections ${ }^{17}$. Our experiments show that the CoV-2 ORF3a protein primes and activates the inflammasome via efflux of potassium ions and the kinase NEK7. Its ability to activate caspase 1, the central mediator of proinflammatory responses, depends on NLRP3 since a selective inhibitor of NLRP3 blocks this pathway in infected cells. Importantly, we find that although the CoV-2 ORF3a protein has diverged somewhat from its homologs in other CoVs, some of these newly divergent residues are essential for activating the NLRP3 inflammasome and are perfectly conserved in virus isolates across continents.

\section{SARS-CoV-2 viroporin ORF3a primes and activates the inflammasome, prompting cell}

\section{death.}

With lung as the predominant site of pathology along with established tropism for kidney and other organs ${ }^{18}$, we introduced ORF3a into lung origin A549 cells and for comparison, kidney origin HEK-293T cells, cell types that readily support SARS-CoV-2 infection ${ }^{19}$, and found induction of pro-IL-1 $\beta$ in both cell types, consistent with priming of the inflammasome. Compared to empty vector-exposed cells, ORF3a also increased the levels of cleaved, i.e. the active form of the proinflammatory caspase, caspase 1 , as well as the cleaved form of the caspase 1 substrate, pro-IL$1 \beta$, indicating activation of the inflammasome, again in both cell types (Fig.1A). Priming by ORF3a resulted from NFKB-mediated expression of $I L-1 \beta$ message (Fig.1B) as indicated by increased I $\kappa \mathrm{B} \alpha$ phosphorylation and enrichment of $\mathrm{NF \kappa B}$ p65 at the $I L-1 \beta$ promoter in ORF3aexposed cells (Figs.1C-E). ORF3a also caused cleavage/activation of Gasdermin D, the pyroptosis-inducing caspase 1-substrate, indicated by an increase in the $\mathrm{N}$-terminal fragment of 
111 Gasdermin D (Fig.1F). This was accompanied by ORF3a-mediated increased cleavage/activation

112 of caspase 3 and cell death, likely secondary to both pyroptosis and apoptosis (Figs.1G and H).

113 Thus, ORF3a primes the inflammasome by triggering NFאB-mediated expression of pro-IL-1 $\beta$

114 while also activating the inflammasome to cleave pro-caspase-1, pro-IL-1 $\beta$, and the pore-forming

115 Gasdermin D, inducing cell death.

modes.

In probing the mechanism of ORF3a-mediated activation of the inflammasome, we found that it enhanced NLRP3 protein levels, and knockdown of NLRP3 curbed ORF3a-directed caspase 1 cleavage (Figs.2A-B), indicating priming and activation of the NLRP3 inflammasome by ORF3a.

122 Further, MCC950, a selective small molecule inhibitor that binds to the NACHT domain of 123 NLRP3 and curtails its activation by blocking ATP hydrolysis ${ }^{20}$, also blocks ORF3a-mediated 124 activation of the inflammasome in low micromolar concentrations (Fig.2C). Moreover, with the 125 NIMA-related kinase NEK7 recently linked to NLRP3 activation ${ }^{16}$, we also depleted NEK7 and 126 found that ORF3a was impaired in its ability to cause cleavage of caspase 1, i.e. unable to activate 127 the inflammasome (Fig.2D). The NLRP3 inflammasome is activated by a variety of cell-extrinsic 128 and -intrinsic stimuli that trigger the assembly of the inflammasome machinery wherein NLRP3 129 oligomerizes with the adaptor protein ASC (Apoptosis-associated speck-like protein containing a 130 CARD) leading to recruitment of pro-caspase 1 which is then activated by proximity-induced 131 intermolecular cleavage. Given ORF3a-mediated inflammasome activation in HEK-293T cells 132 that lack ASC (Fig.2E), we asked if ORF3a activated the inflammasome solely in an ASC- 
independent manner. We found that ORF3a's ability to activate pro-caspase 1 was substantially impaired upon depletion of ASC in A549 cells (Fig.2F), supporting the idea that ORF3a activates the inflammasome in both ASC-dependent and -independent ways. To assess if ORF3a also mediates activation of other prominent inflammasomes including NLRP1 and NLRC4, we depleted each of these molecules but were unable to block cleavage of pro-caspase 1 (Fig.2G), indicating that ORF3a predominantly activates the NLRP3 inflammasome.

\section{ORF3a triggers NLRP3 inflammasome assembly via $\mathrm{K}^{+}$efflux.}

With NEK7 a key mediator of NLRP3 activation downstream of potassium efflux, and efflux of potassium ions a central mechanism of NLRP3 activation, particularly by ion channel-inducing

143 viroporins $15,16,21$, we investigated the effect of blocking potassium efflux by raising the extracellular concentration of $\mathrm{K}^{+}$and found that ORF3a-mediated caspase 1 cleavage was abrogated (Fig.3A). To identify the type of $\mathrm{K}^{+}$channel formed by ORF3a, we employed known pharmacologic inhibitors including quinine, barium, iberiotoxin, and tetraethylammonium to block two-pore domain $\mathrm{K}^{+}$channels, inward-rectifier $\mathrm{K}^{+}$channels, large conductance calcium-activated

$148 \mathrm{~K}^{+}$channels, and voltage gated $\mathrm{K}^{+}$channels, respectively ${ }^{22}$. Mimicking the ability of barium to 149 block the release of SARS-CoV virions ${ }^{23}$ and supporting the finding in Fig.3A, barium was able 150 to curb CoV-2 ORF3a-mediated activation of caspase 1, indicating that ORF3a forms inward151 rectifier $\mathrm{K}^{+}$channels in the cell membrane (Fig.3B). Restricting $\mathrm{K}^{+}$efflux also impaired ORF3a's 152 ability to trigger assembly of both ASC-independent and -dependent NLRP3 inflammasomes 153 (Figs.3C and D, respectively). Notably, not only did SARS-CoV-2 activate the inflammasome 154 upon infection of A549 and HEK-293T cells, but this activation was dampened by MCC950 and 
blocking $\mathrm{K}^{+}$efflux (Figs.3E and F), asserting the importance of ion channels and NLRP3 in triggering the inflammatory response in $\mathrm{CoV}-2$ infected cells.

\section{Key residues in ORF3a important for activating the inflammasome are well conserved.}

Alignment of ORF3a sequences from SARS-CoV-2 isolates from Asia, Europe, Middle-East, Russia, and North and South America between December 2019 and June 2020 as well as other bat CoVs and SARS-CoV revealed the conservation of two out of three key cysteine residues (residues 127, 130, and 133), shown to be essential for $\mathrm{K}^{+}$channel formation by SARS-CoV ${ }^{21}$ (Fig.4A). The exception, cysteine 127, was replaced by leucine in all CoV-2 isolates. We also observed a similar switch from cysteine to valine at position 121 and a switch from asparagine to cysteine at position 153 in all CoV-2 isolates. Introducing single point mutations at positions 127, 130, and 133 of CoV-2 ORF3a impaired its ability to activate the inflammasome, supporting the need for not only the two conserved cysteines at positions 130 and 133 but also that of the newly acquired leucine at position 127 of CoV-2 ORF3a (Fig.4B). Similarly, mutating the residues at positions 121 and 153, both newly acquired in CoV-2 though conserved in all isolates, resulted in a dampened response by the inflammasome (Fig.4B). Thus, SARS-CoV-2 ORF3a has retained some of the key residues needed for virus release and inflammasome activation but it has acquired additional changes that support a functionally consequential divergence from earlier CoVs. Nonetheless, this domain bearing the abovementioned residues that is essential for forming ion channels for virus release has remained remarkably well conserved throughout the pandemic, thereby maintaining its ability to activate the inflammasome. 


\section{Discussion}

178 In summary, an essential viroporin required for release of SARS-CoV-2 from infected cells is also

179 able to prime and activate the NLRP3 inflammasome, the machinery responsible for much of the

180 inflammatory pathology in severely ill patients. ORF3a's indispensability to the virus's life cycle

181 makes it an important therapeutic candidate. Moreover, while different from its homologs in other

182 CoVs, the high conservation of the newly divergent SARS-CoV-2 ORF3a across isolates from several continents combined with our observation that multiple single point mutations reduce its ability to activate the inflammasome, argues against rapid emergence of resistance phenotypes. Thus, targeting ORF3a has the dual potential of blocking virus spread and inflammation.

SARS-CoV-2 is not only linked to severe and fatal outcomes in adults with underlying comorbidities associated with pre-existing inflammation, but it also causes severe disease in children in the form of Multisystem Inflammatory Syndrome in Children (MIS-C) as well as in adults as MIS-A ${ }^{24,25}$. Dampening the inflammatory response in such patients is therefore an attractive strategy - a strategy that has shown promise in a small group of patients treated with Anakinra, a recombinant IL-1R antagonist ${ }^{26}$. Notably, for several inflammatory diseases, there is keen interest within the pharmaceutical industry in therapeutically targeting the inflammatory

194 pathway at a further upstream point, namely NLRP3 itself. MCC950 is a prototype of this approach 195 with several other related compounds undergoing preclinical, phase I, and phase II trials 196 (https://cen.acs.org/pharmaceuticals/drug-discovery/Could-an-NLRP3-inhibitor-be-the-one-drug-

197 to-conquer-common-diseases/98/i7). Along the same lines, Gasdermin D, also activated by 198 ORF3a, presents yet another therapeutic target as it may potentiate virus release by killing cells in 
199 addition to causing inflammation. Additionally, restraining the NLRP3 inflammasome may

200 secondarily stifle virus replication itself as we recently demonstrated for a DNA tumor virus ${ }^{27}$.

202 Aside from ORF3a, other viroporins such as ORF-E and ORF8 may also contribute to the

203 inflammatory response by similar or related mechanisms. Activation of the NLRP3 inflammasome

204 also bears mention in broader contexts. In particular, two recent reports have found that a fraction

205 of severely ill COVID-19 patients display defective type I interferon immunity ${ }^{28,29}$. It is likely that

206 severe disease in these individuals also stemmed from unchecked pro-inflammatory responses

207 since type I interferon can counteract the NLRP3 inflammasome in a number of ways ${ }^{30}$. Similarly,

208 for those who have metabolic disturbances such as hypokalemia that often results from

209 antihypertensive medications, ORF3a may have a lower threshold for activating the inflammasome

210 due to a higher $\mathrm{K}^{+}$gradient across the infected cell.

\section{References}

2131 Liu, Y. et al. Viral dynamics in mild and severe cases of COVID-19. Lancet Infect Dis, 214 doi:10.1016/S1473-3099(20)30232-2 (2020).

2152 Mehta, P. et al. COVID-19: consider cytokine storm syndromes and immunosuppression.

216 Lancet 395, 1033-1034, doi:10.1016/S0140-6736(20)30628-0 (2020).

2173 Jin, Y. \& Fu, J. Novel Insights Into the NLRP 3 Inflammasome in Atherosclerosis. J Am Heart Assoc 8, e012219, doi:10.1161/JAHA.119.012219 (2019). 
2194 Rheinheimer, J., de Souza, B. M., Cardoso, N. S., Bauer, A. C. \& Crispim, D. Current role

220 of the NLRP3 inflammasome on obesity and insulin resistance: A systematic review.

221 Metabolism 74, 1-9, doi:10.1016/j.metabol.2017.06.002 (2017).

2225 Stienstra, R. et al. Inflammasome is a central player in the induction of obesity and insulin

223 resistance. Proc Natl Acad Sci U S A 108, 15324-15329, doi:10.1073/pnas.1100255108

$224 \quad$ (2011).

2256 Chen, W. et al. Activation of the TXNIP/NLRP3 inflammasome pathway contributes to 226 inflammation in diabetic retinopathy: a novel inhibitory effect of minocycline. Inflamm Res $227 \quad 66,157-166$, doi:10.1007/s00011-016-1002-6 (2017).

2287 Dixit, V. D. Nlrp3 inflammasome activation in type 2 diabetes: is it clinically relevant? 229 Diabetes 62, 22-24, doi:10.2337/db12-1115 (2013).

2308 Grant, R. W. \& Dixit, V. D. Mechanisms of disease: inflammasome activation and the 231 development of type 2 diabetes. Front Immunol 4, 50, doi:10.3389/fimmu.2013.00050 232 (2013).

2339 Richardson, S. et al. Presenting Characteristics, Comorbidities, and Outcomes Among 2345700 Patients Hospitalized With COVID-19 in the New York City Area. JAMA, 235 doi:10.1001/jama.2020.6775 (2020).

$23610 \mathrm{Wu}, \mathrm{Z}$. \& McGoogan, J. M. Characteristics of and Important Lessons From the 237 Coronavirus Disease 2019 (COVID-19) Outbreak in China: Summary of a Report of 72314 238 Cases From the Chinese Center for Disease Control and Prevention. JAMA, 239 doi:10.1001/jama.2020.2648 (2020). 
24011 Yang, J. et al. Prevalence of comorbidities and its effects in coronavirus disease 2019 patients: A systematic review and meta-analysis. Int $J$ Infect Dis 94, 91-95, doi:10.1016/j.ijid.2020.03.017 (2020).

24312 Dediego, M. L. et al. Pathogenicity of severe acute respiratory coronavirus deletion mutants in hACE-2 transgenic mice. Virology 376, 379-389, doi:10.1016/j.virol.2008.03.005 (2008).

24613 Ahn, M. et al. Dampened NLRP3-mediated inflammation in bats and implications for a special viral reservoir host. Nat Microbiol 4, 789-799, doi:10.1038/s41564-019-0371-3 (2019).

24914 Ratajczak, M. Z. et al. SARS-CoV-2 Entry Receptor ACE2 Is Expressed on Very Small 250 CD45(-) Precursors of Hematopoietic and Endothelial Cells and in Response to Virus Spike Protein Activates the Nlrp3 Inflammasome. Stem Cell Rev Rep, doi:10.1007/s12015020-10010-z (2020).

25315 Farag, N. S., Breitinger, U., Breitinger, H. G. \& El Azizi, M. A. Viroporins and inflammasomes: A key to understand virus-induced inflammation. Int J Biochem Cell Biol 122, 105738, doi:10.1016/j.biocel.2020.105738 (2020).

25616 He, Y., Zeng, M. Y., Yang, D., Motro, B. \& Nunez, G. NEK7 is an essential mediator of NLRP3 activation downstream of potassium efflux. Nature 530, 354-357, doi:10.1038/nature16959 (2016).

25917 Broz, P. \& Dixit, V. M. Inflammasomes: mechanism of assembly, regulation and 260 signalling. Nat Rev Immunol 16, 407-420, doi:10.1038/nri.2016.58 (2016).

26118 Puelles, V. G. et al. Multiorgan and Renal Tropism of SARS-CoV-2. N Engl J Med 383, 590-592, doi:10.1056/NEJMc2011400 (2020). 
26319 Hoffmann, M. et al. SARS-CoV-2 Cell Entry Depends on ACE2 and TMPRSS2 and Is

264 Blocked by a Clinically Proven Protease Inhibitor. Cell 181, 271-280 e278, 265 doi:10.1016/j.cell.2020.02.052 (2020).

26620 Coll, R. C. et al. MCC950 directly targets the NLRP3 ATP-hydrolysis motif for 267 inflammasome inhibition. Nat Chem Biol 15, 556-559, doi:10.1038/s41589-019-0277-7 268 (2019).

26921 Chen, I. Y., Moriyama, M., Chang, M. F. \& Ichinohe, T. Severe Acute Respiratory 270 Syndrome Coronavirus Viroporin 3a Activates the NLRP3 Inflammasome. Front 271 Microbiol 10, 50, doi:10.3389/fmicb.2019.00050 (2019).

27222 Di, A. et al. The TWIK2 Potassium Efflux Channel in Macrophages Mediates NLRP3 273 Inflammasome-Induced Inflammation. Immunity 49, 56-65 e54, doi:10.1016/j.immuni.2018.04.032 (2018).

27523 Lu, W. et al. Severe acute respiratory syndrome-associated coronavirus 3a protein forms 276 an ion channel and modulates virus release. Proc Natl Acad Sci U S A 103, 12540-12545,

27824 Feldstein, L. R. et al. Multisystem Inflammatory Syndrome in U.S. Children and Adolescents. N Engl J Med 383, 334-346, doi:10.1056/NEJMoa2021680 (2020).

28025 Morris, S. B. et al. Case series of Multisystem Inflammatory Syndrome in Adults 281 associated with SARS-CoV-2 infection - United Kingdom and United States, MarchAugust 2020. Morbidity and Mortality Weekly Report (MMWR) 69, 1450-1456 (2020).

Cavalli, G. et al. Interleukin-1 blockade with high-dose anakinra in patients with COVID19, acute respiratory distress syndrome, and hyperinflammation: a retrospective cohort study. Lancet Rheumatology, doi:https://doi.org/10.1016/S2665-9913(20)30127-2 (2020). 
28627 Burton, E. M., Goldbach-Mansky, R. \& Bhaduri-McIntosh, S. A promiscuous

287 inflammasome sparks replication of a common tumor virus. Proc Natl Acad Sci U S A 117,

288 1722-1730, doi:10.1073/pnas.1919133117 (2020).

28928 Bastard, P. et al. Auto-antibodies against type I IFNs in patients with life-threatening COVID-19. Science, doi:10.1126/science.abd4585 (2020).

29 Zhang, Q. et al. Inborn errors of type I IFN immunity in patients with life-threatening COVID-19. Science, doi:10.1126/science.abd4570 (2020).

30 Labzin, L. I., Lauterbach, M. A. \& Latz, E. Interferons and inflammasomes: Cooperation and counterregulation in disease. $J$ Allergy Clin Immunol 138, 37-46, doi:10.1016/j.jaci.2016.05.010 (2016).

\section{Acknowledgements}

Funding: This research was conducted with funding from the Children's Miracle Network and

299 the University of Florida (S.B.-M).

Author contributions: Conceptualization: H.X., S.B.-M.; Methodology: H.X., M.T.M., S.B.-M.; administration: S.B.-M., Funding acquisition: S.B.-M.

Competing interests: Authors declare no competing interests. materials. 
A

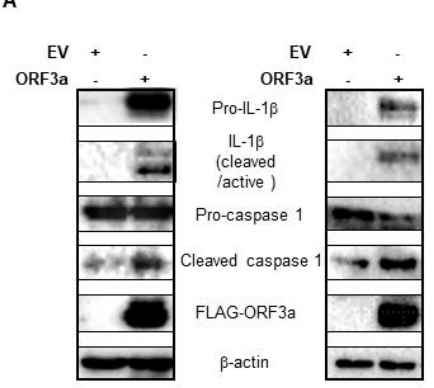

$\mathbf{F}$

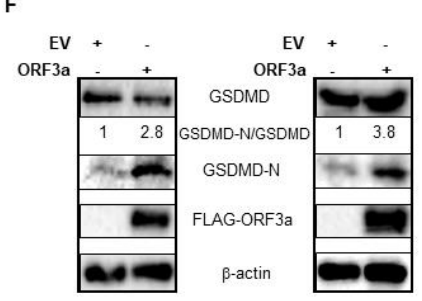

B

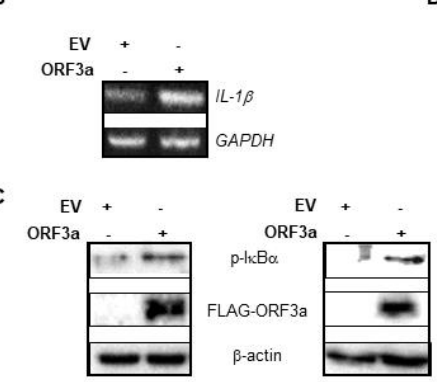

G

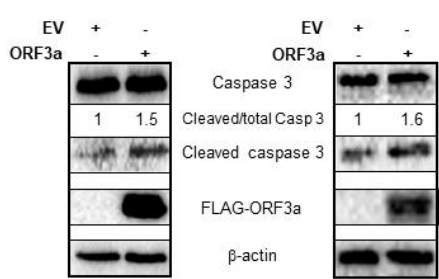

D

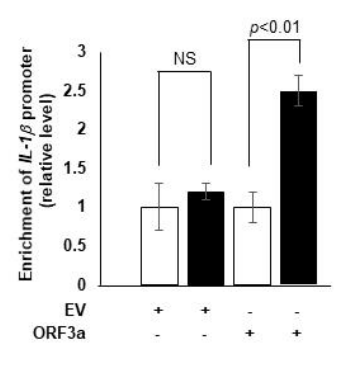

H

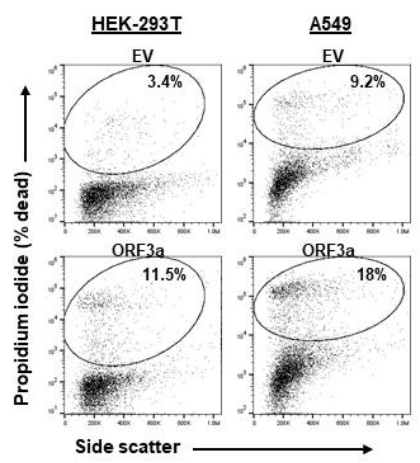
performed three times. 


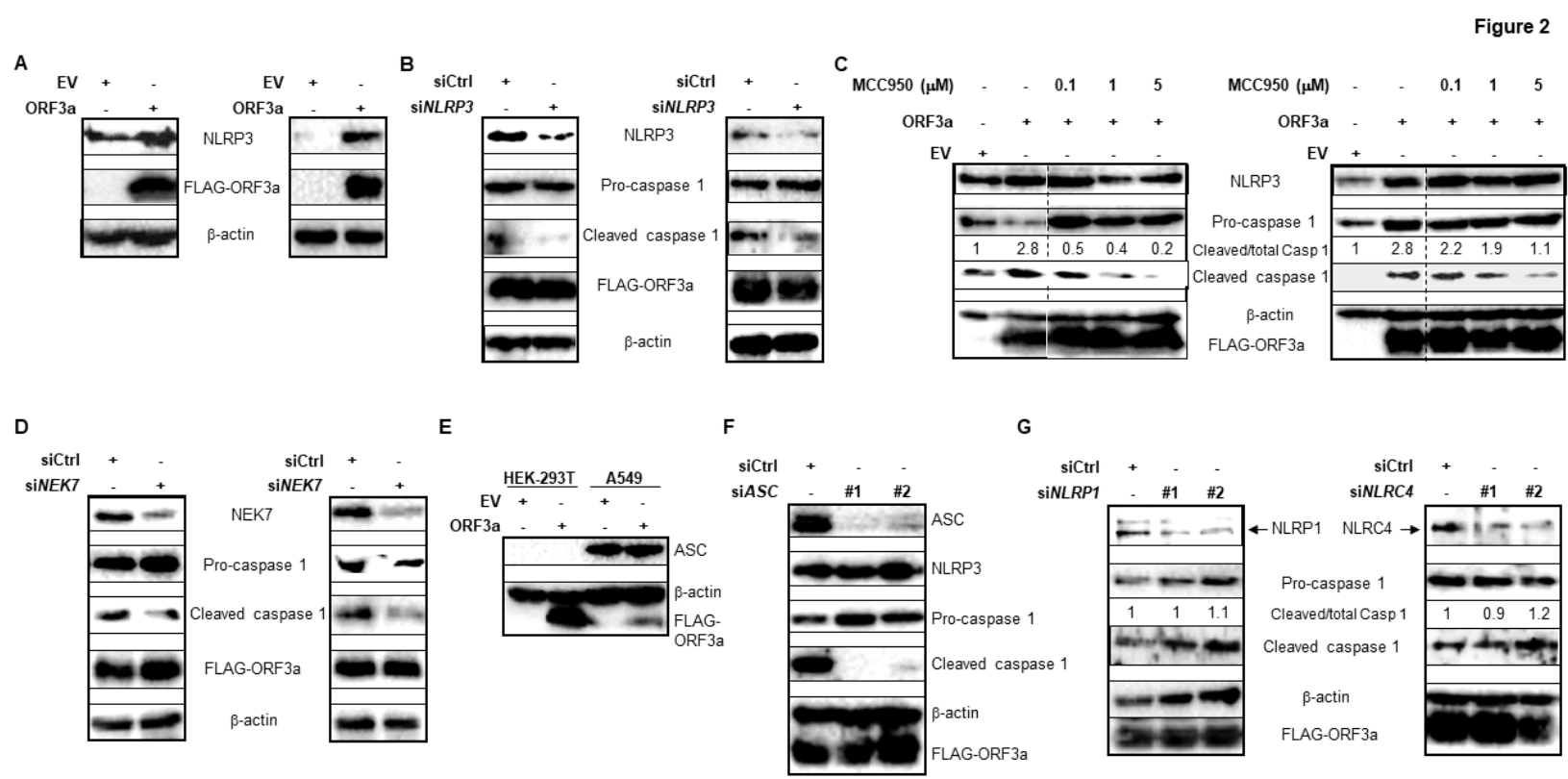
independent modes. (A) Cell lysates of FLAG-ORF3a- or EV-transfected HEK-293T (left) or cells (right) were co-transfected with FLAG- ORF3a and control siRNA (B, D), NLRP3 siRNA $293 \mathrm{~T}$ (left) or A549 cells (right) were transfected with EV or FLAG-ORF3a and exposed to MCC950 for 24 hours prior to immunoblotting. (E) Cell lysates were immunoblotted with indicated antibodies. (F, G) A549 cells were co-transfected with FLAG-ORF3a and control siRNA, $A S C$ siRNA (F), NLRP1 siRNA (G; left), or NLRC4 siRNA (G, right) for 24 hours prior to immunoblotting with indicated antibodies. Experiments were performed at least thrice. 
A

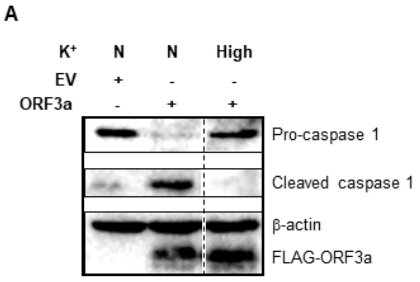

c

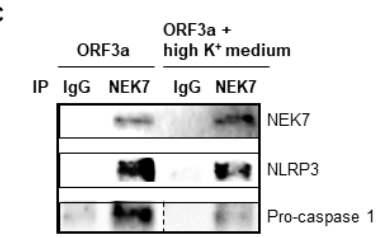

B

Inhibitor

DMSO

ORF3a

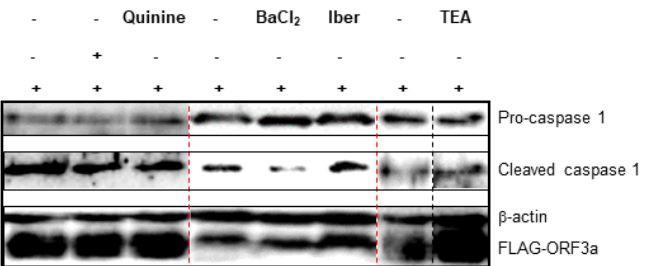

D

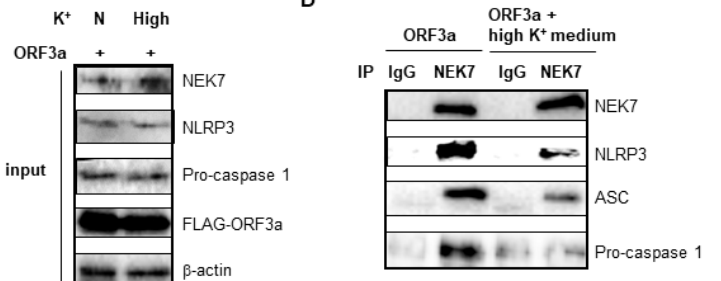

$\mathbf{F}$

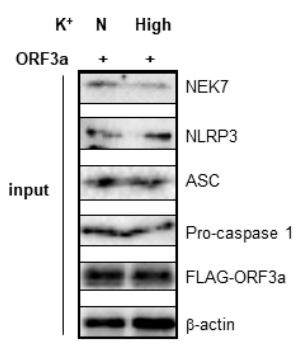

E
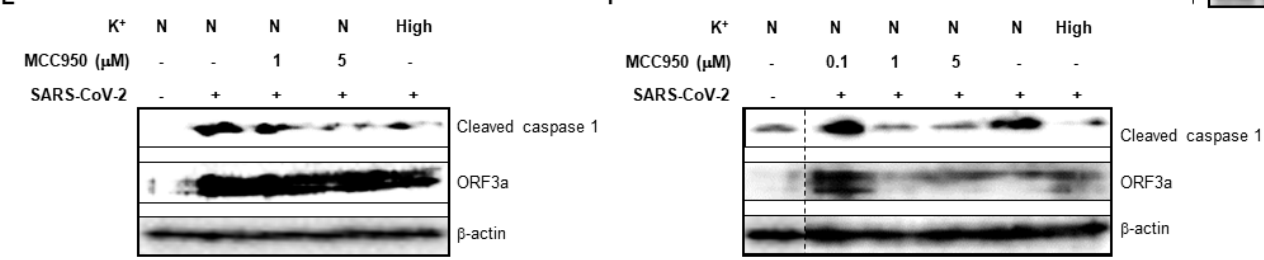

333

334

335

336

337

338

Figure 3. ORF3a-mediated activation of NLRP3 inflammasome requires $\mathrm{K}^{+}$efflux. (A) FLAG-ORF3a plasmid or EV were introduced into A549 cells. After 20 hours, cells were left in normal medium $(\mathrm{N})$ or exposed to medium with high $\mathrm{K}^{+}\left(50 \mathrm{mM}\right.$; to block $\mathrm{K}^{+}$efflux; High). Cells were harvested 4 hours later, and extracts immunoblotted with indicated antibodies. (B) A549 cells transfected with FLAG-ORF3a were exposed to indicated potassium channel inhibitors quinine, barium $(\mathrm{BaCl} 2)$, iberiotoxin (Iber), and tetraethylammonium (TEA) for 24 hours prior to immunoblotting with different antibodies. (C and D) FLAG-ORF3a plasmid was introduced into HEK-293T (C) and A549 (D) cells. After 20 hours, cells were left in normal medium (N) or exposed to medium with high $\mathrm{K}^{+}$(High). Cells were harvested 4 hours later, and extracts immunoblotted (Input) or immunoprecipitated with control IgG or anti-NEK7 antibody followed by immunoblotting with indicated antibodies. Input represents 5\% of sample. (E and F) HEK293T (E) and A549 (F) cells were infected with SARS-CoV-2 in the presence of MCC950 or high $\mathrm{K}^{+}$ 

immunoblotting with indicated antibodies. Experiments were performed twice.

A
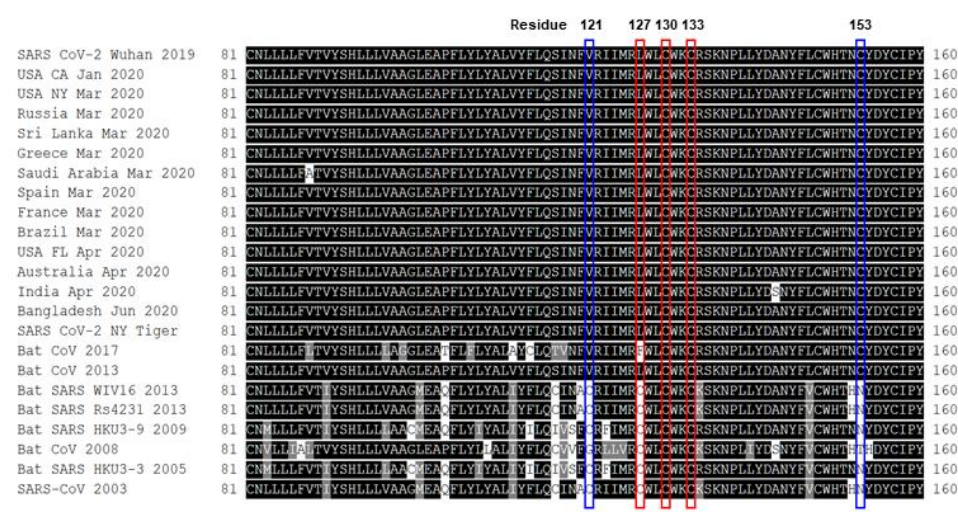

B

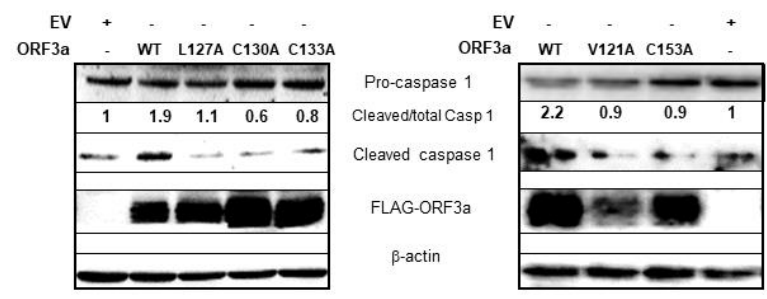

Figure 4

Figure 4. ORF3a residues required for inflammasome activation are conserved in SARS-

354 pandemic and diverse species isolates dating back to the original SARS pandemic of 2003 were the most diversity are shown from positions 81 to 160 of ORF3a/ORF3. Conserved cysteine residues previously identified in SARS-CoV as critical to $\mathrm{K}^{+}$ion channel formation are outlined in red. Newly divergent residues (121 and 153) conserved across SARS-CoV-2 isolates are 
360 outlined in blue. The multiple sequence alignment was shaded in BoxShade hosted by ExPASy

361 (https://embnet.vital-it.ch/software/BOX_form.html). (B) A549 cells were transfected with EV,

362 wild-type FLAG-ORF3a (WT), or FLAG-ORF3a mutants. Cells were harvested 24 hours later and

363 immunoblotted with indicated antibodies. Experiments were performed at least twice. 
bioRxiv preprint doi: https://doi.org/10.1101/2020.10.27.357731; this version posted October 27, 2020. The copyright holder for this preprint (which was not certified by peer review) is the author/funder, who has granted bioRxiv a license to display the preprint in perpetuity. It is made

available under aCC-BY-NC-ND 4.0 International license.

1 Methods

\section{$2 \quad$ Cell lines and infection}

3 Human embryonic kidney-293T (HEK-293T) cells were maintained in DMEM

4 (Thermo Fisher Scientific, Cat. 11965118) containing 10\% fetal bovine serum 5 (GEMINI, Cat. 900108) and 1\% penicillin/streptomycin (Gibco, Cat. 15140122). A549

6 cells were maintained in Ham's F-12 Nutrient Mix (Thermo Fisher Scientific, Cat.

7 11765054) containing 10\% fetal bovine serum and 1\% penicillin/streptomycin. Both

8 cell lines were cultured in the presence of $5 \% \mathrm{CO} 2$ at $37^{\circ} \mathrm{C}$. Cells were infected in a

9 BSL-3 lab with the UF-1 strain of SARS-CoV-2 at MOI of 4 in media containing 3\% low IgG FBS (Fisher Scientific, Cat. SH30070.03).

Plasmids, siRNAs, and transfection

The ORF3a gene without stop codon (nt 25,382-26,206, GenBank accession no.

MT295464.1) was PCR amplified with forward primer (5'CGCGGATCCATGGATTTGTTTATGAGAATCTT3') and reverse primer (5' AAGGAAAAAAGCGGCCGCCAAAGGCACGCTAGTAGTC3') by using Phusion

High-Fidelity DNA Polymerase (New England Biolabs, M0530L) according to the manufacturer's protocol and inserted into pcDNA5.1/FRT/TO vector (a kind gift from professor Torben Heick Jensen, Denmark) with a C-terminal $3 \times$ FLAG tag to generate FLAG-tagged ORF3a plasmid. Flag-tagged ORF3a mutants (V121A, L127A, C130A,

21 C133A, C153A) were constructed by overlap extension PCR with the following primer pairs: 
bioRxiv preprint doi: https://doi.org/10.1101/2020.10.27.357731; this version posted October 27, 2020. The copyright holder for this preprint (which was not certified by peer review) is the author/funder, who has granted bioRxiv a license to display the preprint in perpetuity. It is made

5'GAGTATAAACTTTGCAAGAATAATAATGAG3' (forward) and

5'CTCATTATTATTCTTGCAAAGTTTATACTC3' (reverse),

5'ATAATGAGGGCTTGGCTTTG3' (forward) and

5'CAAAGCCAAGCCCTCATTAT3' (reverse),

5'GCTTTGGCTTGCCTGGAAATGC3' (forward) and

5'GCATTTCCAGGCAAGCCAAAGC3' (reverse),

5'TGCTGGAAAGCCCGTTCCAAA3' (forward) and

5’TTTGGAACGGGCTTTCCAGCA3' (reverse),

5'GCATACTAATGCTTACGACTATTG3' (forward) and

5'CAATAGTCGTAAGCATTAGTATGC3' (reverse), respectively.

HEK-293T and A549 cells were transfected with LipoJet ${ }^{\mathrm{TM}}$ In Vitro Transfection Kit

(SignaGen Laboratories, SL100468) according to the manufacturer's protocol.

HEK-293T and A549 cells were transfected with 200 pmoles of siRNA. siRNAs included NLRP3 (Ambion, Cat. s41554), NEK7 (Ambion, Cat. 103794), ASC (Ambion, Cat. 44232 and 289672), NLRP1 (\#1, Ambion, Cat. S22520; \#2, Ambion, Cat. 239345), NLRC4 ((\#1, Ambion, Cat. S33828; \#2, Ambion, Cat. 105219), and control (Dharmacon, Cat. D001810-01-20).

\section{SARS-CoV-2}

A passage two stock of Severe acute respiratory syndrome coronavirus 2 isolate SARSCoV-2/human/USA/UF-1/2020 (GenBank MT295464) was used for virus-infection studies. The virus was the first isolate from a patient at the University of Florida Health 
bioRxiv preprint doi: https://doi.org/10.1101/2020.10.27.357731; this version posted October 27, 2020. The copyright holder for this preprint (which was not certified by peer review) is the author/funder, who has granted bioRxiv a license to display the preprint in perpetuity. It is made

45 Shands Hospital (J. Lednicky, unpublished) and has about $99 \%$ nt identity with SARS-

CoV-2 reference strain Wuhan-Hu-1 (GenBank NC_045512.2) and 100\% identity with the genomes of SARS-CoV-2 detected in California, USA. The genome of SARS-CoV2 UF-1 encodes an aspartic acid residue at amino acid 614 of the spike protein. This virus was isolated and then propagated (one passage) in VeroE6 cells prior to sequence analyses and use in this work, and has no INDELs in its genome. All work with this virus was performed in a BSL-3 laboratory by an analyst using a full-head powered-air purifying respirator and appropriate personal protective equipment, including gloves and a chemically impervious Tyvek gown.

\section{Chemical treatment of cell lines}

HEK-293T and A549 cells were transfected with plasmids. After 2h, different chemical reagents were added to medium. Chemical reagents included NLRP3 inhibitor MCC950 (0.1-5 $\mu \mathrm{M})$ (Sigma Aldrich, Cat. 538120), Quinine (10 $\mu \mathrm{M})$ (Sigma Aldrich, Cat. 145904), Barium chloride (2 mM) (Sigma Aldrich, Cat. 342920), Tetraethylammonium chloride (5 mM) (Tocris Bioscience, Cat. 306850), and Iberiotoxin $(1.0 \mu \mathrm{M})$ (Tocris Bioscience, Cat. 1086100U). All chemicals were dissolved with DMSO or sterile water.

\section{Reverse transcription PCR (RT-PCR)}

RT-PCR was performed as previously described ${ }^{1}$. Briefly, $1 \mu \mathrm{g}$ of total RNA was used as template for complementary DNA synthesis using MuLV reverse transcriptase (New 
bioRxiv preprint doi: https://doi.org/10.1101/2020.10.27.357731; this version posted October 27, 2020. The copyright holder for this preprint (which was not certified by peer review) is the author/funder, who has granted bioRxiv a license to display the preprint in perpetuity. It is made available under aCC-BY-NC-ND 4.0 International license. England Biolabs, Cat. M0253L) according to the manufacture's protocol. OneTaq DNA Polymerase (New England Biolabs, Cat. M0480S) was used to amplify DNA fragment using manufacture's protocol. RT-PCR primers were as following:

forward primer 5'ACCATCTTCCAGGAGCGAGA3' and

reverse primer 5'GGCCATCCACAGTCTTCTGG 3' for GAPDH mRNA, forward primer 5'TCAGCCAATCTTCATTGCTC3' and reverse primer 5'GCCATCAGCTTCAAAGAACA3' for $I L-1 \beta$ pre-mRNA ${ }^{2}$.

\section{Immunoblotting and antibodies}

Immunoblotting was performed as previously described ${ }^{3}$. Briefly, total cell lysates were electrophoresed on $10 \%$ or $12 \%$ SDS-polyacrylamide gels and transferred onto nitrocellulose membranes and immunoassayed with indicated antibodies. The following antibodies were used: rabbit anti-Caspase-1 antibody (Thermo Scientific, Cat. PA587536), rabbit anti-cleaved Caspase-1 antibody (Thermo Scientific, Cat. PA538099), mouse anti-IL-1 $\beta$ (Cell Signaling Technology, Cat. 12242s), mouse antiFlag M2 antibody (Sigma-Aldrich, Cat. F1804), mouse anti- $\beta$-actin antibody (Sigma-

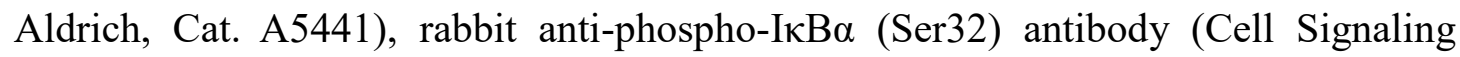
Technology, Cat. 2859s), rabbit anti-IкB $\alpha$ antibody (Cell Signaling Technology, Cat. 9242s), rabbit anti-NF-кB p65 antibody (Cell Signaling Technology, Cat. 8242s), rabbit anti-Caspase 3 antibody (GeneTex, Cat. GTX110543), rabbit anti-Gasdermin D (L60) antibody (Cell Signaling Technology, Cat. 93709s), rabbit anti-cleaved-Gasdermin D (Asp275) antibody (Cell Signaling Technology, Cat. 36425s), rabbit anti-NLRP3 
bioRxiv preprint doi: https://doi.org/10.1101/2020.10.27.357731; this version posted October 27, 2020. The copyright holder for this preprint (which was not certified by peer review) is the author/funder, who has granted bioRxiv a license to display the preprint in perpetuity. It is made available under aCC-BY-NC-ND 4.0 International license.

89 antibody (Invitrogen, Cat. PA5-21745), rabbit anti-NEK7 antibody (Cell Signaling

90 Technology, Cat. 3057s), rabbit anti-ASC antibody (Cell Signaling Technology, Cat.

91 13833s), rabbit anti-NLRP1 antibody (Novus Biologicals, Cat. NB100-56147SS), rabbit anti-NLRC4 antibody (Novus Biologicals, Cat. NB100-56142SS), rabbit antiSARS-CoV-2 ORF3a antibody (FabGennix, Cat. SARS-COV2-ORF3A-101AP), HRPconjugated goat anti-mouse $\operatorname{IgG}(\mathrm{H}+\mathrm{L})$ (Thermo Scientific, Cat. 626520) and HRP conjugated goat anti-rabbit $\operatorname{IgG}(\mathrm{H}+\mathrm{L})$ (Thermo Scientific, Cat. 31460), and HRP conjugated goat anti-rabbit IgG (light chain) (Novus, Cat. NBP2-75935).

\section{Flow cytometry}

Flow cytometry was performed as previous described ${ }^{4}$. Briefly, HEK-293T and A549 cells were treated with trypsin for 3 min and collected by centrifugation at $350 \mathrm{~g}$ for 3 min. Cell pellets were washed twice with FACS buffer (1X PBS with 2\% FBS) and resuspended in $200 \mu \mathrm{l}$ of RNase-containing FACS buffer. $20 \mu \mathrm{l}$ of propidium iodide (10 $\mu \mathrm{g} / \mathrm{ml}$ ) (Sigma-Aldrich, Cat. P4864) was added to each sample and subjected to flow cytometry immediately to assay cell death.

\section{Chromatin immunoprecipitation-quantitative PCR (ChIP-qPCR)}

ChIP was performed as described previously ${ }^{5}$. Briefly, A549 cells were transfected with FLAG-ORF3a or empty vector as control. Twenty-four hours later, cells $\left(7.5 \times 10^{5}\right.$ cells for each ChIP) were crosslinked with $1 \%$ formaldehyde for $20 \mathrm{~min}$ and quenched with $0.125 \mathrm{M}$ glycine. Cells were lysed in $500 \mu \mathrm{l}$ of nuclear extraction buffer A (Cell 
bioRxiv preprint doi: https://doi.org/10.1101/2020.10.27.357731; this version posted October 27, 2020. The copyright holder for this preprint (which was not certified by peer review) is the author/funder, who has granted bioRxiv a license to display the preprint in perpetuity. It is made available under aCC-BY-NC-ND 4.0 International license.

111 Signaling Technology, Cat. 7006) on ice for $15 \mathrm{~min}$ and washed once with $500 \mu \mathrm{l}$ of nuclear extraction buffer B (Cell Signaling Technology, Cat. 7007), and then treated with $0.5 \mu \mathrm{L}$ of micrococcal nuclease (Cell Signaling Technology, Cat. 10011) for 20 min at $37^{\circ} \mathrm{C}$. Nuclei were resuspended in $1 \times$ ChIP buffer (Cell Signaling Technology, Cat. 7008) and sonicated at $8 \mathrm{~W}$ with 10 -s on and 20-s off pulses on ice for two cycles to break nuclear membranes. After removing debris, $2 \%$ of each sample was set aside as input and the rest (98\%) of the sample was incubated with $3 \mu \mathrm{g}$ of antibody (or $3 \mu \mathrm{g}$ of IgG as control) and $30 \mu \mathrm{l}$ of protein G magnetic beads (Cell Signaling Technology, Cat. 7008) at $4{ }^{\circ} \mathrm{C}$ overnight. Beads were washed three times with low salt ChIP buffer and once with high salt ChIP buffer. The protein-DNA complex were eluted with $1 \times$ Elution buffer (Cell Signaling Technology, Cat. 10009). DNA was extracted with DNA purification columns (Cell Signaling Technology, Cat. 10010) and subjected to qPCR analysis. The following primers were used for amplifying the $I L-1 \beta$ promoter: forward primer 5'AGGAGTAGCAAACTATGACAC3' and reverse primer 5'ACGTGGGAAAATCCAGTATTT3' 6 .

\section{Co-Immunoprecipitation (Co-IP)}

Co-IP was performed as described previously ${ }^{7}$. Cells were lysed in ice-cold IP Lysis Buffer (Thermo Scientific, Cat. 87787) in the presence of $1 \times$ protease inhibitor cocktail (Cell Signaling, \#7012) for $15 \mathrm{~min}$ followed by centrifugation $(14,000 \mathrm{rpm})$ at $4{ }^{\circ} \mathrm{C}$ for $5 \mathrm{~min}$. Of pre-cleared cell lysates, $5 \%$ was set aside as input. The rest was incubated with $3.0 \mu \mathrm{g}$ of rabbit anti-NEK7 antibody (Bethyl Laboratories, Cat. A302-684A) or 
bioRxiv preprint doi: https://doi.org/10.1101/2020.10.27.357731; this version posted October 27, 2020. The copyright holder for this preprint (which was not certified by peer review) is the author/funder, who has granted bioRxiv a license to display the preprint in perpetuity. It is made

133 the same amount of control IgG (R\&D, Cat. AB-105-C) together with $40 \mu \mathrm{l}$ of

134 Dynabeads Protein G (Thermo Scientific, Cat. 10003D) at $4{ }^{\circ} \mathrm{C}$ overnight. Beads were

135 washed three times with IP lysis buffer and subjected to immunoblotting.

\section{Statistical analysis}

Unpaired Student's t test was used to calculate $p$ values by comparing the means of two

139 groups.

\section{References}

1421 King, C. A., Li, X., Barbachano-Guerrero, A. \& Bhaduri-McIntosh, S. STAT3

2 Siu, K. L. et al. Severe acute respiratory syndrome coronavirus ORF3a protein activates the NLRP3 inflammasome by promoting TRAF3-dependent ubiquitination of ASC. FASEB J 33, 8865-8877, doi:10.1096/fj.201802418R (2019).

3 Burton, E. M., Goldbach-Mansky, R. \& Bhaduri-McIntosh, S. A promiscuous

15, e1008228, doi:10.1371/journal.ppat.1008228 (2019). 
bioRxiv preprint doi: https://doi.org/10.1101/2020.10.27.357731; this version posted October 27, 2020. The copyright holder for this preprint (which was not certified by peer review) is the author/funder, who has granted bioRxiv a license to display the preprint in perpetuity. It is made available under aCC-BY-NC-ND 4.0 International license.

1555 Li, X. et al. KRAB-ZFP Repressors Enforce Quiescence of Oncogenic Human

156 Herpesviruses. J Virol 92, doi:10.1128/JVI.00298-18 (2018).

1576 Hiscott, J. et al. Characterization of a functional NF-kappa B site in the human

158 interleukin 1 beta promoter: evidence for a positive autoregulatory loop. Mol

159 Cell Biol 13, 6231-6240, doi:10.1128/mcb.13.10.6231 (1993).

1607 Li, X., Burton, E. M. \& Bhaduri-McIntosh, S. Chloroquine triggers Epstein-

161 Barr virus replication through phosphorylation of KAP1/TRIM28 in Burkitt

162 lymphoma cells. PLoS Pathog 13, e1006249,

163 doi:10.1371/journal.ppat.1006249 (2017). 